\title{
WRR4 Encodes a TIR-NB-LRR Protein That Confers Broad-Spectrum White Rust Resistance in Arabidopsis thaliana to Four Physiological Races of Albugo candida
}

\author{
M. Hossein Borhan, ${ }^{1}$ Nick Gunn, ${ }^{2}$ Abigail Cooper, ${ }^{2}$ Sigrun Gulden, ${ }^{1}$ Mahmut Tör, ${ }^{2}$ S. Roger Rimmer, ${ }^{1}$ \\ and Eric B. Holub' \\ ${ }^{1}$ Agriculture and Agri-Food Canada, Saskatoon Research Centre, Saskatoon, SK., S7N 0X2, Canada; ${ }^{2}$ University \\ of Warwick, Warwick-HRI, Wellesbourne, Warwickshire, CV35 9EF, U.K.
}

Submitted 4 December 2007. Accepted 28 February 2008.

White blister rust in the Brassicaceae is emerging as a superb model for exploring how plant biodiversity has channeled speciation of biotrophic parasites. The causal agents of white rust across a wide breadth of cruciferous hosts currently are named as variants of a single oomycete species, Albugo candida. The most notable examples include a major group of physiological races that each are economically destructive in a different vegetable or oilseed crop of Brassica juncea (A. candida race 2), B. rapa (race 7), or B. oleracea (race 9); or parasitic on wild crucifers such as Capsella bursa-pastoris (race 4). Arabidopsis thaliana is innately immune to these races of $A$. candida under natural conditions; however, it commonly hosts its own molecularly distinct subspecies of $A$. candida (A. candida subsp. arabidopsis). In the laboratory, we have identified several accessions of Arabidopsis thaliana (e.g.,. Ws-3) that can permit varying degrees of rust development following inoculation with $A$. candida races 2,4 , and 7 , whereas race 9 is universally incompatible in Arabidopsis thaliana and nonrusting resistance is the most prevalent outcome of interactions with the other races. Subtle variation in resistance phenotypes is evident, observed initially with an isolate of $A$. candida race 4 , indicating additional genetic variation. Therefore, we used the race 4 isolate for mapbased cloning of the first of many expected white rust resistance $(W R R)$ genes. This gene was designated WRR4 and encodes a cytoplasmic toll-interleukin receptor-like nucleotide-binding leucine-rich repeat receptor-like protein that confers a dominant, broad-spectrum white rust resistance in the Arabidopsis thaliana accession Columbia to representative isolates of $A$. candida races $2,4,7$, and 9, as verified by transgenic expression of the Columbia allele in Ws-3. The WRR4 protein requires functional expression of the lipase-like protein EDS1 but not the paralogous protein PAD4, and confers full immunity that masks an underlying nonhypersensitive incompatibility in Columbia to $A$. candida race 4 . This residual incompatibility is independent of functional EDS1.

M. Hossein Borhan and Nick Gunn were joint first authors who contributed equally to the research.

Corresponding authors: E. B. Holub; E-mail: eric.holub@warwick.ac.uk; and M. H. Bohran; E-mail: borhanh@agr.gc.ca
Additional keywords: basidiomycete, gene-for-gene, innate immunity, Leptosphaeria, natural variation, nonhost, $R B, B s 2$, RLM1, sid2.

Arabidopsis thaliana (L.) Heynh. has been used extensively for molecular genetic investigations of innate immunity to infection by bacterial, viral, fungal, and oomycete pathogens under laboratory conditions (Buell 1998; Crute et al. 1994; Glazebrook 2001). Most of the microorganisms used in past research were collected as pathogens of various crops. Important exceptions include two oomycetes that are common parasites of Arabidopsis thaliana, at least in western Europe (Holub and Beynon 1997): the downy mildew pathogen Hyaloperonospora arabidopsis (formerly Peronospora parasitica) (Göker et al. 2004), and a subspecies of the white blister rust pathogen Albugo candida (Pers. ex. Fr.) Kuntze (referred to hence as A. candida subsp. arabidopsis). A. candida subsp. arabidopsis is molecularly divergent from the predominant group of physiological races in A. candida (Fig. 1) (Choi et al. 2006; Rehmany et al. 2000; Voglmayr and Riethmüller 2006). These races are closely related but each is economically destructive in a different vegetable or oilseed crop of Brassica juncea (A. candida race 2), B. rapa (race 7), or B. oleracea (race 9); or parasitic on wild crucifers such as Capsella bursapastoris (race 4) (Liu et al. 1989; Pound and Williams 1963; Verma et al. 1975).

Extensive natural variation exists in Arabidopsis thaliana for downy mildew resistance to $H$. arabidopsis, thus providing a rich resource for the rapid identification in a small number of accessions of more than 20 genes, each of which specifies resistance to a different combination of $H$. arabidopsis isolates (Holub 2007; Holub and Beynon 1997; Slusarenko and Schlaich 2003). At least six of these so-called "recognition of $P$. parasitica" (RPP) genes have been molecularly characterized (Holub $2001)$ and, like the largest class of plant resistance $(R)$ genes, all encode receptor-like proteins that have a nucleotide-binding (NB) site and a highly variable C-terminal leucine-rich repeat (LRR) domain. Plant NB-LRR genes have been further subdivided by $\mathrm{N}$-terminal sequence as either coiled-coil (CC)-NBLRR containing a coiled-coil domain, or toll-interleukin receptor-like (TIR)-NB-LRR containing a TIR domain (for similarity to drosophila toll and mammalian interleukin-1 receptor) (Jones and Jones 1997). Interestingly, RPP genes have been characterized from both of these NB-LRR subclasses (Holub 2001). 
The LRR domain of pathogen receptor-like proteins is generally thought to be associated with the specific recognition of a matching pathogen elicitor (Hammond-Kosack and Jones 1997). Distinct modes of induced defense, which exhibit an imperfect correspondence with subclasses of NB-LRR genes (distinguished by either an N-terminal TIR-like or CC domain), have been revealed from mutational analyses of bacterial and downy mildew resistance in Arabidopsis thaliana (Aarts et al. 1998; McDowell et al. 2000; Shapiro 2000; Tör et al. 2002). In addition, a "gene-for-gene" relationship was established in 2002 for five molecularly characterized $R P P$ genes and their corresponding specific elicitors in $H$. arabidopsis, referred to as genes encoding Arabidopsis thaliana-recognized (ATR) effectors, and two of these ATR genes have since been molecularly characterized (Holub in press). However, the nature of protein-protein interactions in $R P P$-mediated recognition of $H$. arabidopsis has yet to be elucidated.
In stark contrast, minimal natural variation exists in Arabidopsis thaliana for white rust resistance to Albugo candida subsp. arabidopsis. Based on visual assessment, this disease is more prevalent than downy mildew in wild U.K. populations of Arabidopsis thaliana, which may be explained largely by the low frequency (less than 10\%) of Albugo candida subsp. arabidopsis resistance in U.K. and global collections of Arabidopsis thaliana (Holub in press; Holub et al. 1995). All of the standard accessions commonly used in laboratory research are fully susceptible to Albugo candida subsp. arabidopsis. Interestingly, genetically dominant hypersensitive cell death is a rare phenotype among the nonstandard resistant accessions. In most cases, the incompatibility retards growth of the parasite and restricts rust development with associated symptoms of chlorosis or loss of turgidity in the immediate vicinity of colonized tissue (Holub et al. 1995). Three specificities for A. candida subsp. arabidopsis resistance have been genetically characterized, and

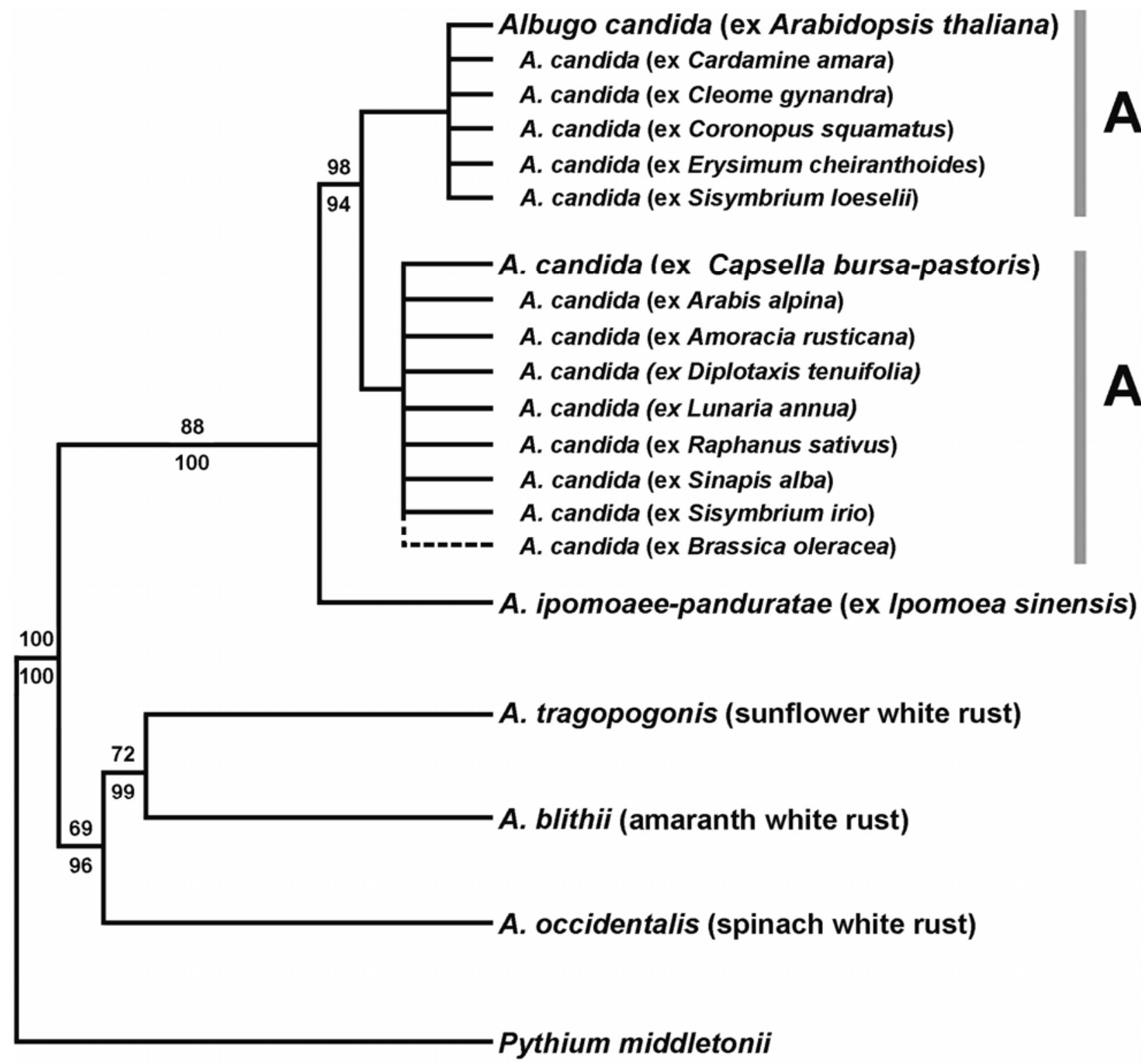

Fig. 1. Molecular evidence for two subgroups within Albugo candida that are labeled here as AcA (typified by isolates collected from Arabidopsis thaliana) and AcB (typified by Albugo candida race 4 from Capsella bursa-pastoris). The phylogenetic relationship between these subgroups was described by Voglmayr and Reithmüller (2006), who compared nuclear large-subunit ribosomal DNA sequences from 60 isolates of Albugo spp. in a Bayesian analysis (using Markov chain Monte Carlo analysis over five million generations). An isolate from a brassica host (dashed line) was not included in their study; however, additional data from amplified fragment length polymorphism and internal transcribed spacer 1 sequence comparisons indicate that brassica-derived isolates (including races 2, 7, and 9 from Brassica juncea, B. rapa, and B. oleracea, respectively) are grouped with AcB race 4 (Choi et al. 2006; Rehmany et al. 2000; N. Gunn and E. B. Holub, unpublished). 
recognition of $A$. candida (RACl) was molecularly characterized as a gene encoding a TIR-NB-LRR protein (Borhan et al. 2001, 2004).

The circumstances are reversed in the case of the predominant group of $A$. candida races that have evolved as specialized parasites of other crucifer hosts. Under natural conditions, Arabidopsis thaliana appears to be innately immune as a species to this Albugo candida subgroup, including races 2, 4, 7, and 9. In laboratory experiments, $A$. candida race 9 is universally avirulent in U.K. and global collections of Arabidopsis thaliana (N. Gunn and E. B. Holub, unpublished). However, we have identified several accessions (e.g., Ws-3) that can permit varying degrees of rust development following inoculation with representative isolates of Albugo candida races 2,4 , and 7 . Using careful assessment of the variation in resistance phenotypes, we report the genetic mapping in an available recombinant inbred population (derived from a cross between two resistant accessions Columbia and Niederzenz), along with mutational analyses, that enabled molecular characterization of a gene that encodes a cytoplasmic TIR-NB-LRR receptor-like protein. The effect of several Arabidopsis thaliana defense mutants on white rust resistance 4 (WRR4) also was examined.

\section{RESULTS}

\section{Phenotypic characterization}

of wild-type resistance to Albugo candida subsp. B.

Three laboratory accessions of Arabidopsis thaliana (Columbia glabrous-1 [Col-gll], Niederzenz [Nd-1], and Wassilewskija [Ws-3]) illustrate the full range of interaction phenotypes following inoculation of cotyledons with representative isolates of four Albugo candida races, including race 2 collected from B. juncea (Ac2) race 4 from $C$. bursa-pastoris (Acem2), race 7 from B. rapa (Ac7), and race 9 from B. oleracea (Ac9 'Stratford').

Col-gll exhibited full immunity to all four of the A. candida isolates (Fig. 2). Cotyledons retained a green appearance similar to noninoculated tissue with no formation of white blisters 6 to 10 days after inoculation (dai). Necrotic flecks were barely visible because the parasite typically arrested in development at the primary vesicle stage (1 dai) as it formed in the underside of an epidermal cell lying at the roof of a stomatal chamber, following growth of the germ tube through the stomatal aperture. This arrested development of the parasite was associated with hypersensitive response of the penetrated epidermal cell, as indicated by trypan blue and diaminobenzedene (DAB) staining (Fig. 3A through $\mathrm{C}$ ), and callose encasement of the primary vesicle (not shown) by 3 dai.

In contrast, Ws-3 exhibited compatibility with three of the A. candida isolates. Acem 2 was the most aggressive isolate, producing white blisters profusely on the lower surface of cotyledons and often on the upper surface at 6 to 10 dai (Fig. 2 ). This isolate could readily progress into the juvenile leaves and cause severe stunting from the systemic infection. No cellular host response was visible microscopically following DAB treatment (not shown). Similarly, isolates Ac2 and Ac7 were readily capable of producing white blisters on either the upper or lower surfaces of inoculated cotyledons, also without visible host response (Fig. 2). However, the degree of rusting with these isolates was markedly less and inconsistent among inoculated cotyledons compared with Acem 2 inoculations. The isolate Ac9 Stratford was least aggressive, being able to form restricted colonies in the mesophyll without hypersensitive host cell death (necrotic flecks) but consistently unable to produce even minute blisters (Fig. 2). These combined observations indicate that Ws-3 was a suitable accession for transformation experiments to test candidate $R$ genes for their ability to confer white rust resistance to any of the four $A$. candida isolates.

Intermediate incompatibility of all four A. candida isolates was observed in Nd-1. Acem2, Ac2, and Ac7 infrequently produced small blisters 6 to 10 dai, usually on the upper surface of cotyledons (Fig. 2). The surrounding host tissue became flaccid following inoculation with the more aggressive isolate Acem 2 and exhibited a visible change to gray or chlorotic appearance. Extensive hyphal growth was observed 3 dai with no apparent host cell death (not shown). The interaction phenotype observed following inoculation of $\mathrm{Nd}-1$ with the race 9 isolate was similar to inoculations of Ws-3 with the same isolate.

\section{Genetic mapping of WRR4.}

The full immunity observed in Col-0 (referred to hence as the green cotyledons with no blisters [GN] phenotype) was chosen as a suitable target for map-based cloning of a gene that is capable of conferring this resistance phenotype. Given our primary interest in testing whether the resistance was broad spectrum against different races of $A$. candida, we required comparative tests of the same host genotypes with each of the A. candida isolates and, thus, chose to use an $\mathrm{F}_{9}$ Colglabrous 1 (Col-gll) $\times \mathrm{Nd}-1$ inbred population that had been generated previously for genetic analyses of downy mildew resistance (Holub and Beynon 1997). An $\mathrm{F}_{2}$ Col-gll $\times \mathrm{Nd}-1$ population was inoculated initially with Acem 2 to assess the genetic complexity of rust resistance in this cross. The GN phenotype was observed in 238 of $309 \mathrm{~F}_{2}$ seedlings. None of the remaining seedlings exhibited transgressive segregation for full (Ws-type) susceptibility but did vary in degrees of flaccidity, with or without chlorosis. This suggested that a single dominant gene (238:71 GN:non-GN; $\left.\chi^{2}=0.67, P=0.41\right)$ conferred the full immunity phenotype in Col-gll, and that this resistance masked a residual incompatibility (nonhypersensitive, flaccid response) that includes at least one gene that is shared between Col-gll and $\mathrm{Nd}-1$ or resistance conferred by two very tightly linked genes.

These genetic predictions were supported by phenotypic assessment of $187 \mathrm{~F}_{9}$ recombinant inbreds from Col- $g l l \times \mathrm{Nd}$ 1 following inoculations with Acem2. Again, none of these inbreds exhibited transgressive segregation for full susceptibility, and 89 (approximately 50\%) were fully immune, similar to Col-gll, indicating that a single gene is conferring this resistance in the segregating population (89:98 GN:non-GN; $\chi^{2}=$ $0.43, P=0.51)$. Molecular markers that were already available from the recombinant inbred population were used to identify a locus on the lower arm of chromosome one, approximately 8 centimorgans on the telomeric side of the simple sequence length polymorphism (SSLP) marker nga280. This locus was designated WRR4; the previous "RAC" abbreviation was not used in this case to avoid further confusion with GTPase genes in Arabidopsis thaliana that are referred to by the same abbreviation.

Inoculations of the same recombinant inbreds with Ac2 and Ac7 revealed $100 \%$ correlation of GN versus non-GN resistance phenotypes that had been observed with Acem2 inoculations. This indicated that, indeed, the WRR4 locus was associated with resistance to at least three races of Albugo candida.

\section{Cloning of WRR4.}

Additional molecular markers were used to refine the mapping of WRR4 to an interval delimited by NG-8 and HB-1 (Fig. 4A). This region contains three predicted TIR-NB-LRR receptor-like genes (At1g56510, At1g56520, and At1g56540), which became obvious candidates for transformation experiments. 
Two bacterial artificial chromosomes (BAC) (F13N6 and F25P12) spanning the WRR4 region were subcloned in the binary cosmid vector SLJ75515, and polymerase chain reaction (PCR) primer pairs designed from intergenic sequences between each of the candidate genes were used to identify representative cosmid subclones. The terminal sequences of three cosmid clones (B5, C4, and $\mathrm{P} 18$ ) verified their relative positions in the WRR4 interval (Fig. 4A). The A. candida-susceptible accession Ws-3 then was transformed with each clone, and transgenic plants $\left(\mathrm{T}_{1}\right)$ were selected for resistance to the herbicide

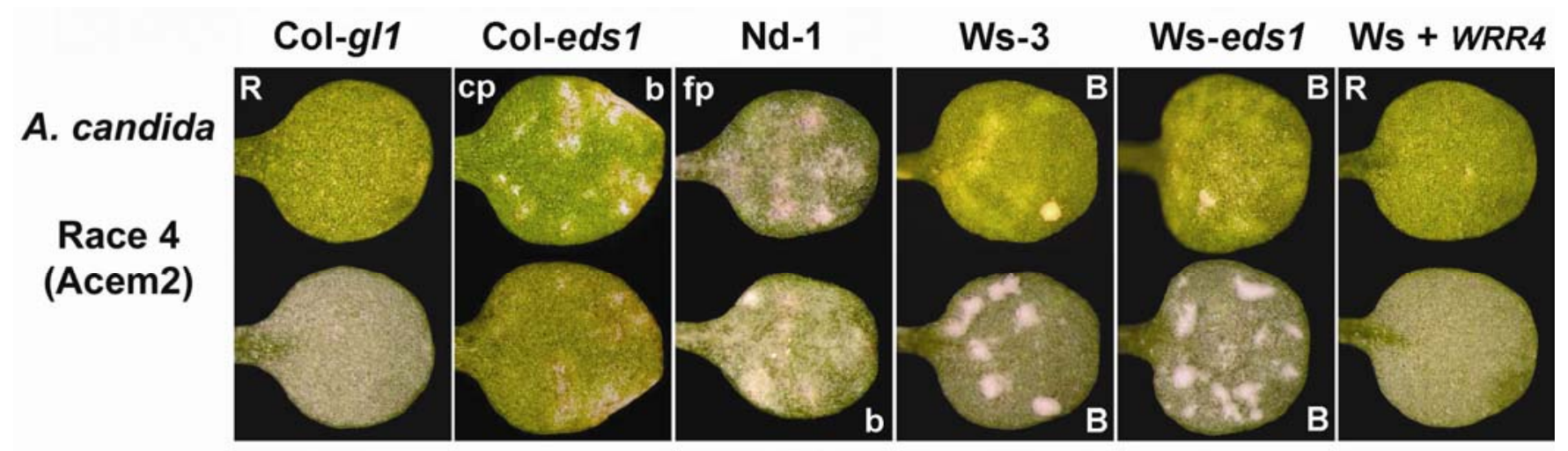

Race 2
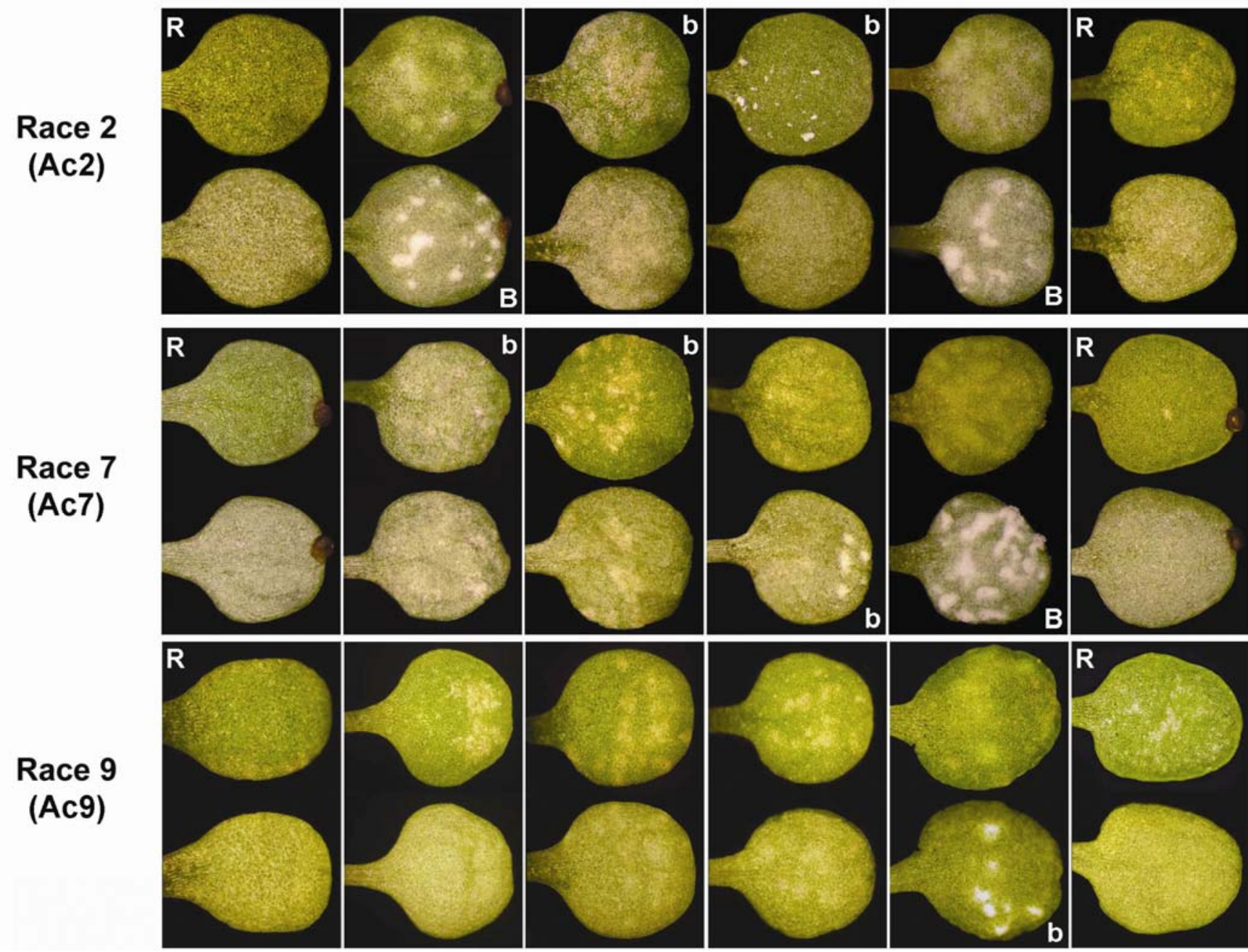

Fig. 2. Interaction phenotypes of wild-type and mutant Arabidopsis thaliana inoculated with zoospores of Albugo candida isolates representing four physiological races, including race 2 collected from Brassica juncea (Ac2), race 4 from Capsella bursa-pastoris (Acem2), race 7 from B. rapa (Ac7), and race 9 from B. oleracea (Ac9). The upper and lower cotyledon surfaces are shown for each combination of host and parasite. The laboratory accession Columbia glabrous-1 (Col-gll) exhibited full immunity to all four isolates, with no macroscopic symptoms but rapid host cell death visible microscopically that arrested parasite development at the primary vesicle stage (phenotype labeled by white "R"). Wild-type Wassilewskija (Ws-3) was fully susceptible to Acem2 (race 4), permitting development of large white blisters without visible host response (labeled by "B"); partially susceptible to Ac2 and Ac7, permitting small blisters (labeled by "b"); and incompatible to Ac9, with no blisters forming from small colonies that formed in the mesophyll without visible host response. Mutation of the lipase-like gene EDS1 enhanced compatibility to all four isolates. Wild-type Niederzenz (Nd-1) exhibited intermediate phenotypes with each isolate. Acem 2 caused a host response in Nd-1 that was visible as a flaccid patch (indicated by "fp"), which contrasted with a chlorotic patch ("cp") response in Col-eds I (an RNAi suppression line). The white rust resistance gene WRR4 from Col-0 conferred a full immunity phenotype to all four isolates when expressed transgenically in Ws-3 (Ws + WRR4, far right column). 

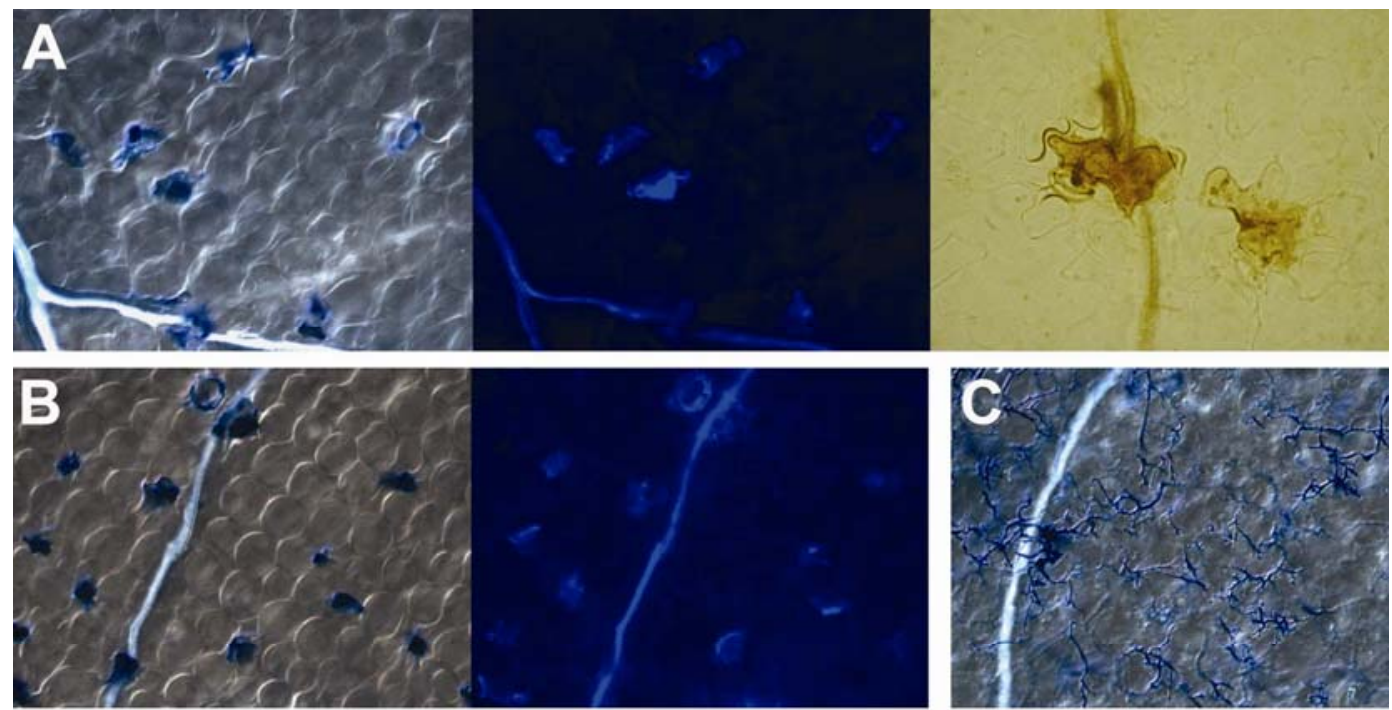

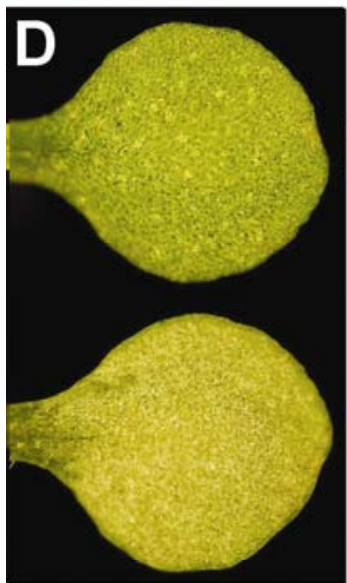

Col-gl1

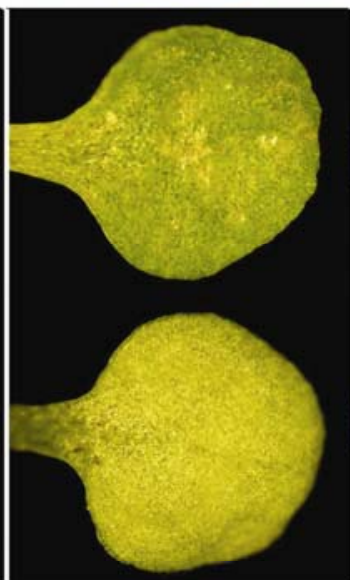

Ws + P18

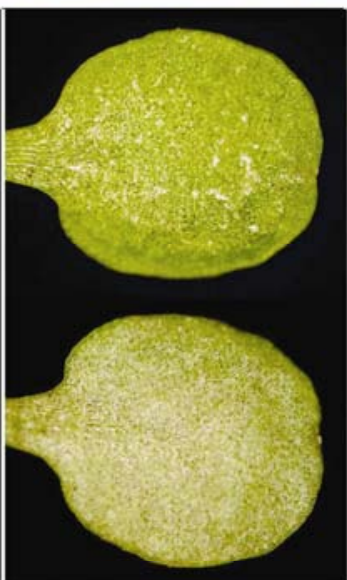

Ws + B5

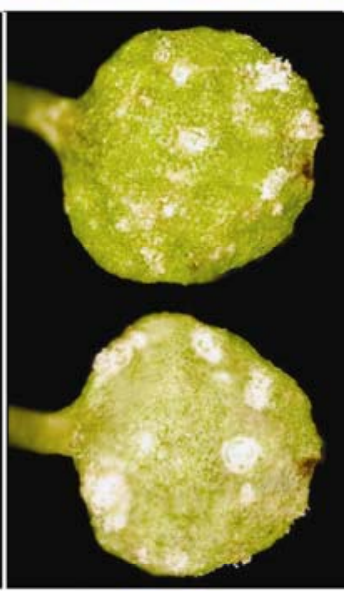

$\mathrm{Ws}+\mathrm{C} 4$

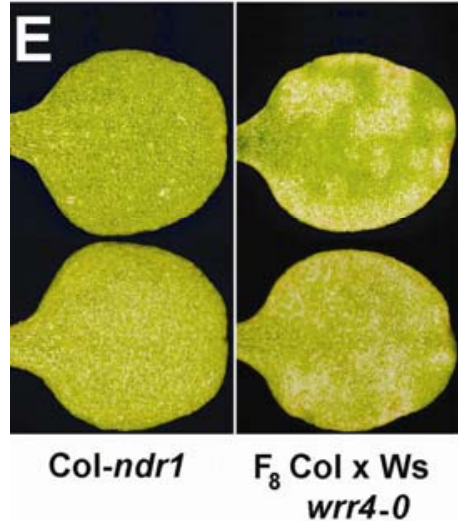

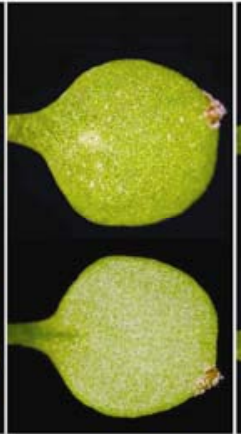

Col-wrr4-1 (EMS)

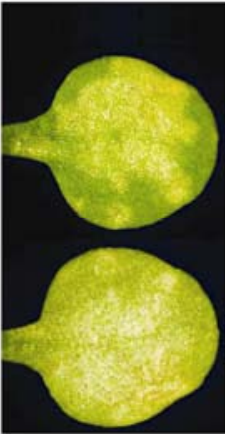

Col-ndr1 wrr4-2 (FN)

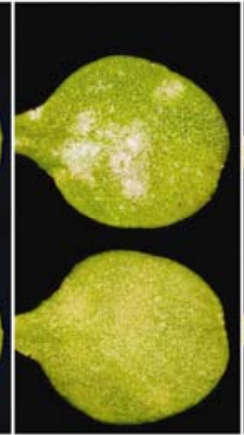

$F_{1}$ NDR1/ndr1 wrr4-1/wrr4-2

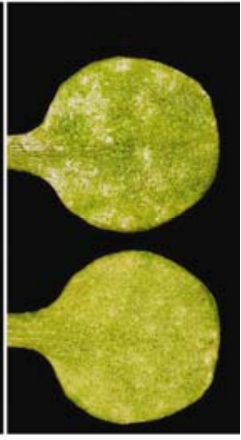

Col-wrr4-4

(TI)

Fig. 3. Photographs illustrating parasite development and host phenotypes of wild-type, mutant. and transgenic Arabidopsis thaliana following inoculation with physiological races of Albugo candida collected from other crucifer species. A through $\mathbf{C}$, Race 2, isolate Ac2 from Brassica juncea; D and E, race 4, isolate Acem2 from Capsella bursa-pastoris. The parasite initiates infection by inserting its germ tube into the stomatal chamber and producing its primary vesicle in the underside of a single epidermal cell (Holub et al. 1995). A, Wild-type white rust resistance (WRR) gene, WRR4, resistance in Col-gll consistently causes the parasite to arrest development in the first epidermal cell by eliciting a hypersensitive response that is visible with the accumulation of autofluroscent material (left photo, trypan blue-stained cells visible with white light; middle photo, same cells fluorescing with UV excitation) and oxidative burst (right photo, visible by diaminobenzedene staining of the penetrated epidermal cell and the parasite germ tube. B, White rust resistance similar to wildtype in Wassilewskija (Ws-3) transformed with a subclone (P18) from genomic Columbia DNA containing WRR4 (left photo, white light illumination; right photo, UV excitation). C, Profuse sporulation of A. candida (blue-stained hyphae) in Ws-3 transformed with a subclone (C4) that contains a nonfunctional homolog of WRR4 from Columbia. D, Interaction phenotypes of Ws-3 transformed with cosmid P18 (resistant), cosmid B5 (resistant), and cosmid C4 (susceptible). E, The wrr4 mutations exhibiting enhanced susceptibility (reduced resistance), including a fast-neutron mutant, Col-ndrl/wrr4-2, with distinct chlorotic patches of colonized tissue similar to the phenotype observed in a representative F8 ColxWs-wrr4-0 inbred; a TDNA-insertion mutant, Col-wrr4-4, with a similar degree of enhanced susceptibility; and an EMS mutant, Col-wrr4-1, with less-pronounced patches. The lack of Acem2 sporulation in all three mutants indicates that additional genes confer resistance to this parasite in Col-gll. The Col-ndrl background for the fast-neutron mutant was fully immune, similar to wild-type Columbia, indicating the NDR1 is not required for WRR4-mediated resistance, whereas the chlorotic patch phenotype of the F1 Ndr1/ndr1, wrr4-1/wrr4-2 phenotype confirms allelism of the two WRR4 mutants. 

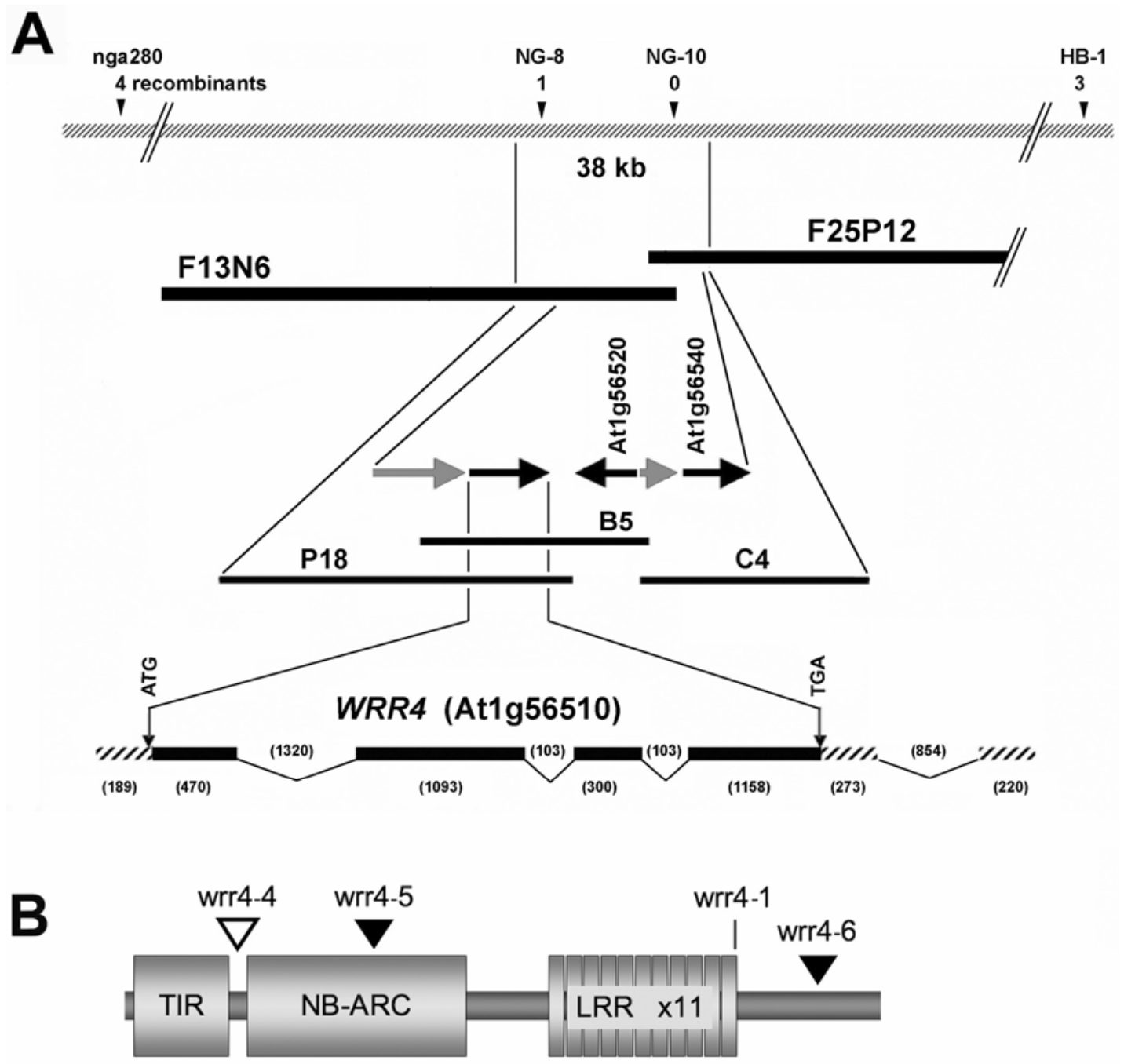

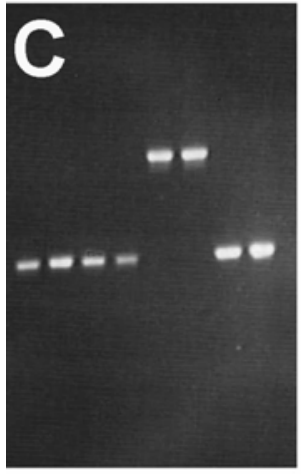

WRR4

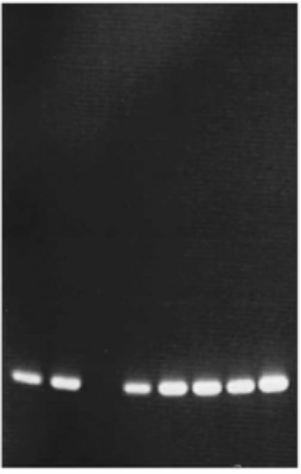

At1g56520

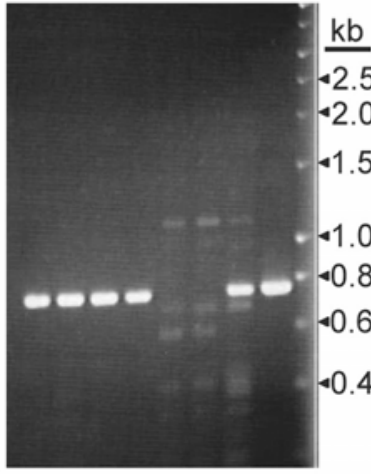

At1g56540

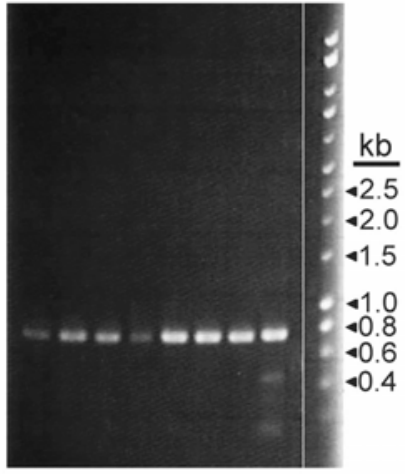

At1g56550

Fig. 4. Map-based cloning of WRR4 from Arabidopsis thaliana Columbia and supporting evidence from fast-neutron mutants. A, Position of white rust resistance (WRR) gene, WRR4, (At1g56510) on chromosome 1 (the hatched bar) and position of some of the markers used for mapping WRR4 indicated by arrow. The bacterial artificial chromosomes spanning across the WRR4 map contig are shown as solid bars. Cosmid clones spanning across this region are shown by solid lines. Location of exons (solid bars), introns (broken lines), and untranslated regions (hatched bars) are depicted in the lowest bar diagram. B, Diagram of the WRR4 including N-terminal Toll-interleukin receptor-like domain (TIR), nucleotide-binding ARC domain (NB-ARC), and 11 leucine rich-repeats (LRRs). The C-terminus is leucine rich but without an repeat structure. Mutations are indicated above, including wrr4-1 (EMS), which caused a single amino acid change in the last LRR; and TDNA insertion mutants in the first intron (wrr4-4, open triangle) in the NB domain (wrr4-5, black triangle indicates exon insertion) and non-LRR C-terminal region (wrr4-6, exon insertion). C, Gene-specific polymerase chain reaction products were used to detect genetic rearrangements in two fast neutron mutants (wrr4-2 and wrr4-3) that affected WRR4 and a paralogous gene At1g56540, but not middle paralog (At1g56520) or the next telomeric gene (At1g56550). The bands were generated from the following DNA sources (left to right): Col-gll (WRR4), Col-0 (WRR4), Ws-0 (wrr4-0), Col-ndr1 (WRR4), Col-ndr1/wrr4-2, Col-ndr1/wrr4-3, Col-wrr4-1, and the appropriate BAC clones (F13N6 or F25P12). 
DL-phosphinothricin (PPT). The $\mathrm{T}_{2}$ of lines that contained either B5 or P18 segregated approximately 3:1 for GN versus non-GN phenotypes following inoculation with either Ac2 or Acem2. All of the non-GN seedlings were herbicide sensitive following PPT treatment. $\mathrm{T}_{3}$ lines that were homozygous for B5 or P18 were uniformly resistant to all four A. candida isolates, including the race 9 isolate (Figs. 2 and 3D, representative photographs from Acem 2 inoculations). In contrast, no $A$. candida resistance was observed in $\mathrm{T}_{2}$ or $\mathrm{T}_{3}$ lines containing the C4 cosmid. At1g56510 is the only full-length TIR-NBLRR gene that is contained on both B5 and P18, indicating that this is most likely the gene responsible for conferring WRR4-mediated resistance.

To confirm the reported sequence of At1g56510, we sequenced the WRR4 gene from cosmid P18 using gene-specific primers and found no differences. Alignment with genomic sequence revealed a gene that contains three introns within the coding region, and a $3^{\prime}$ untranslated region (UTR) of $490 \mathrm{bp}$ separated by an intron of $854 \mathrm{bp}$ (Fig. 4A). The predicted WRR4 protein consists of 1,077 amino acids (aa) (molecular mass of $113.9 \mathrm{kDa}$ ) containing a TIR domain, an NB domain, 11 LRR of variable length based on consensus sequence described by Jones and Jones (1997), and a nonLRR tail of 125 aa.

\section{Mutational analyses confirms the TIR-NB-LRR identity of WRR4.}

More than 50,000 $\mathrm{M}_{2}$ seedlings (derived from EMS or fastneutron-treated $\mathrm{M}_{1}$ parents) were screened for enhanced susceptibility to Acem2. None were found which permitted development of white blisters. However, one ethane methyl sulphonate (EMS) mutant of Col-gll and two fast-neutron mutants of Col-ndrl were identified which permitted small colonies of Acem 2 to develop in the mesophyll, visible macroscopically as discrete brown or chlorotic patches 7 to 10 dai (Fig. 3E). All three of these mutants did permit Ac2 or Ac7 to produce white blisters without a visible host response, indicating that a single $R$ gene (WRR4) confers resistance in Col-0 to these $A$. candida isolates.

The EMS mutant (Col-gll/wrr4-1) has a single nucleotide base change in genomic DNA of At1g56510 at position 4,543 (transcribed position 2,509) causing a predicted amino acid change from a cysteine to a tyrosine (amino acid position 837). This mutation is within the $\beta$-strand/ $\beta$-turn region (xLxLxxC) of the last (eleventh) LRR (Fig. 4B). The two fast-neutron mutants (Col-ndrl/wrr4-2 and Col-ndrl/wrr4-3) appear to have the same chromosomal rearrangement that alters the PCR amplified products of both At1g56510 and At1g56540 (Fig. 4C). At1g56540 already had been ruled out as a candidate gene because the cosmid clone $\mathrm{C} 4$ (which solely contains this TIRNB-LRR copy) did not confer A. candida resistance.

Three T-DNA insertional knockout mutants of At1g56510 also were examined, including Salk_013292 (insertion in intron one; designated Col-wrr4-4), Salk_148037 (insertion in exon two within the predicted NB site; Col-wrr4-5), and Salk_131774 (insertion in exon 4 within the non-LRR segment; Col-wrr4.6) (Fig. 4B). All three of these mutants exhibited pronounced chlorotic patches following inoculation with Acem 2 (Fig. 3E) and formation of blisters with Ac2 and Ac7 inoculations (not shown). These phenotypes were similar to those observed in the fast-neutron mutants, indicating that the $n d r l$ mutation contributed no discernable enhanced susceptibility.

The combined mutational evidence supports the conclusion from the transgenic experiments described above that At1g56510 is a single gene at the WRR4 locus which confers broad-spectrum, full immunity to several races of $A$. candida.
The effect of eds1 mutation on WRR4-mediated resistance.

As with other TIR-NB-LRR disease resistance proteins, the WRR4-mediated resistance appears to require functional expression of the lipase-like gene EDS1 (Aarts et al. 1998). This was evident from enhanced susceptibility of a Col-eds 1 RNAi line following inoculation with each of the A. candida isolates (Fig. 2). No blistering was observed following inoculation with Acem 2 or Ac9, indicating that the residual resistance to A. candida races 4 and 9 is $E D S 1$ independent. Interestingly, this resistance is naturally variable in Arabidopsis thaliana because the nonhost resistance to Albugo candida race 9 in the Ws- 0 background is fully dependent on EDS1 (Parker et al. 1996). Similarly, enhanced formation of white blisters was observed in our study following inoculations of Ws-eds 1 with Ac2 and Ac7 (Fig. 2 ), suggesting that Ws-3 also contains a low level or basal incompatibility to A. candida races 2 and 7 which is EDS1 dependent.

\section{The effect of other defense signaling mutations on WRR4-mediated resistance.}

We attempted to determine whether other defense regulatory mutants also affected WRR4-mediated resistance. As described above, $n d r l$ does not appear to alter the WRR4 resistance phenotype (Fig. 3E). Similarly, other mutations in a Columbia background that exhibit reduced levels of salicylic acid (SA) also had no macroscopic impact on WRR4 resistance following inoculation with Acem2, Ac2, or Ac7. These mutations included fmol, npr1, pad4.1, sgt1b, rarl/pbs2, sid1, sid2, and the transgenic line Col-NahG. Microscopically, however, hyphal growth appeared to extend further in sid1, sid2, and $\mathrm{rarl} / \mathrm{pbs} 2$ following inoculation with Ac2 or Ac7 (penetration of up to six mesophyll cells in these mutants, compared with cessation in the first host cell in wild-type Col-0) (data not shown).

\section{DISCUSSION}

We have characterized a gene designated WRR4 that encodes a cytoplasmic TIR-NB-LRR receptor-like protein in Columbia Arabidopsis thaliana and confers broad-spectrum white rust resistance to four physiological races of Albugo candida described by Pound and Williams (1963) and Liu and associates (1989). Under natural conditions, Arabidopsis thaliana appears to be innately immune as a species to these four Albugo candida races, which instead thrive on their preferred hosts, including different brassica crops and other wild crucifers such as $C$. bursa-pastoris. All four races belong to a predominant group of $A$. candida that is molecularly distinct from the subspecies referred to here as A. candida subsp. arabidopsis, which commonly is found in the United Kingdom, causing white rust in natural Arabidopsis thaliana populations (Fig. 1) (Choi et al, 2006; Rehmany et al. 2000; Voglmayr and Riethmüller 2006). In this context, WRR4 is similar to other single $R$ genes that have previously been described including $R B$ in the wild potato relative Solanum bulbocastanum and $B s 2$ in pepper that confer broad spectrum disease resistance to late blight or bacterial speck respectively (Tai et al. 1999; Song et al. 2003).

WRR4 (At1g56510) was mapped to a 40-kb region on chromosome 1 containing two additional genes that encode TIRNB-LRR proteins (At1g56520 and At1g56540). Transgenic evidence with subclones from this region and supporting mutant analysis indicated that only one gene (At1g56510) was able to confer white rust resistance. A BLAST search of the Arabidopsis thaliana protein database identified the neighboring genes as being the most similar to WRR4 (74 and $76 \%$ similarity, respectively). Meyers and associates (2003) previously had grouped all three genes in the same clade (TNL-H) 
of NB-LRR genes in Arabidopsis thaliana Col-0. Interestingly, this clade also includes the stem canker resistance gene $R L M 1$ (At1g64070; 54\% identity and 64\% similarity with WRR4) described by Staal and associates (2006), whereas RPPl-Ws is the most closely related downy mildew resistance gene $(41 \%$ identity and 59\% similarity). Sequence analysis of the wrr $4-1$ EMS mutant allele revealed an amino acid change of $\mathrm{C}$ (cysteine) to $\mathrm{Y}$ (tyrosine) at position 837 . The point mutation lies within the $\beta$-strand/ $\beta$-turn motif of the last LRR of WRR4 (Jones and Jones 1997). Comparison of the solvent exposed $\beta$ strand/ $\beta$-turn structure within the LRR domain of other known $R$ genes shows that this motif is hypervariable and under diversifying selection (Bittner-Eddy et al. 2000; Botella et al. 1998; McDowell et al. 1998; Meyers et al. 1998). As with these previous examples, it is plausible that the LRR of WRR4 is important for recognition specificity of this novel WRR protein.

WRR4 is structurally similar to another TIR-NB-LRR gene called $R A C 1$ that previously was characterized as a gene conferring Albugo candida subsp. arabidopsis resistance in Arabidopsis thaliana (Borhan et al. 1995). As with other examples of TIR-NB-LRR disease resistance genes, RACl and WRR4 each require the EDS1 lipase-like protein to confer resistance (Aarts et al. 1998; Feys et al. 2001; Peart et al. 2002). However, unlike the other examples, both of the WRR genes appear to function independently from a second lipase-like protein, PAD4. In addition, WRR4 appears to function independently from FMO1 which is a positive regulator of the EDS1 defense pathway in Arabidopsis thaliana and required for bacterial and downy mildew resistance conferred by other TIR-NB-LRR proteins (Bartsch et al. 2006).

Inducible defense responses involving EDS1 in Arabidopsis thaliana generally have been correlated with SA activity (Wiermer et al. 2005). Enhanced hyphal development of $\mathrm{Al}$ bugo candida in two mutants (sidl and sid2) may suggest at least a partial role for constitutive levels of SA in regulating WRR4-mediated resistance because these genes are involved in biosynthesis of SA. In an analogous study, Mellersh and Heath (2003) conducted mutational analyses of nonhost resistance in Arabidopsis thaliana to the basidiomycete rust pathogen from cowpea, Uromyces vignae. They concluded that SA plays an important role in restricting compatibility of basidiomycete rusts in Arabidopsis thaliana, as indicated by enhanced hyphal growth and haustorial development of $U$. vignae in the sid2 mutant, whereas SA-dependent expression of pathogenesis-related proteins (indicative of hypersensitive defense responses) provides no significant contribution to the resistance.

A role for SA in WRR4-mediated resistance, however, does not appear to involve the elevated expression of the $\mathrm{R}$ protein itself in Columbia Arabidopsis thaliana. Tan and associates (2007) reported that SA treatment of leaf tissue significantly elevated levels of TIR-NB-LRR proteins that are known to confer downy mildew resistance in wild-type Columbia. However, they observed that constitutive expression of WRR4 was among the lowest compared with other known NB-LRR resistance proteins in nonelicited tissue, with the highest expression of WRR4 in leaf tissue and approximately threefold lower expression in flowers and siliques; and that the effect of SA treatment on WRR4 expression was negligible. Interestingly, the relationship between SA signaling and WRR4 expression may vary markedly in different genetic backgrounds because a sevenfold increase in WRR4 expression was detected in the Libyan accession Mt-0 following treatment of leaf tissue with SA (Tan et al. 2007).

SA is an important molecule for signaling stomatal closure to restrict the entry of motile epiphytic bacteria into the stomatal chamber (Underwood et al. 2007). Guard cells can detect the presence of these bacteria and signal SA-mediated closure via membrane receptors such as FLS2, an LRR receptor-like kinase that detects the nonspecific elicitor protein flagellin (Gomez-Gomez and Boller 2000; Melotto et al. 2006). Albugo candida and many basidiomycete rusts such as $U$. vignae also typically attempt to invade a potential host via stomata. Thus, a recent report by Zipfel and associates (2004) seems particularly fascinating. They used microarray experiments for assessing the response of leaf tissue to flg22 treatment (a fragment of bacterial flagellin protein), and detected an increased expression of WRR4 (approximately sixfold) in wild-type Landsberg erecta. A similar flg22-elicited response was observed with the WRR4-paralog At1g56540, whereas most other TIR-NB-LRR proteins were unaffected in expression. Some virulent bacterial pathogens release a phytotoxin coronatine that can suppress the stomotal closure process mediated by FLS2 and SA (Melotto et al. 2006).

A distinction in signaling attributes among members of the TIR-NB-LRR subfamily will be an important factor to investigate for its potential role in determining host specialization among subspecies of $A$. candida. For instance, basic compatibility of A. candida subsp. arabidopsis in Arabidopsis thaliana involves broad-spectrum suppression of programmed cell death and innate immunity to other biotrophs (Cooper et al. 2008), including suppression of downy mildew resistance conferred by several TIR-NB-LRR genes. However, RACl and $W R R 4$ represent important exceptions to this phenomenon as receptor-like $R$ genes in Arabidopsis thaliana that can induce defense in a manner that is nonsuppressible by the corresponding isolates of Albugo candida. This suggests that they differ intrinsically from other TIR-NB-LRR genes, and particularly ones that confer downy mildew resistance, either in timing with an ability to induce a more rapid defense response that preempts defense suppression or perhaps in the biochemistry of the defense signaling process (e.g., PAD4-dependent defense is suppressible whereas PAD4-independent defense of $R A C 1$ and WRR4 may be nonsuppressible by preinfection with A. candida subsp. arabidopsis). The latter prediction could be tested by sequential inoculation of transgenic Arabidopsis thaliana Ws-WRR4, first with a virulent isolate of Albugo candida subsp. arabidopsis and subsequently with an avirulent isolate of $A$. candida (race 2, 4, or 7), similar to the experiments described by Cooper and associates (2008).

The WRR4 phenotype in Col-gll does exhibit characteristics of a rapid, full immunity to at least four physiological races of A. candida. No symptoms were visible macroscopically and parasite development invariably was arrested in the first host cell. A rapidly induced host response was evident because an oxidative burst was detected by the accumulation of hydrogen peroxide (shown by DAB staining) following inoculation of Col-gll with the A. candida race 4 isolate Acem2. In contrast, the much slower, less-potent attenuation of white rust development in $\mathrm{Nd}-1$ did not appear to involve a rapid oxidative burst indicative of host cell death. Interestingly, colonization of $\mathrm{Nd}$ 1 by Acem 2 was associated with a loss of turgidity, which was not evident with the other three A. candida isolates; and Ac9 was strictly unable to reproduce in $\mathrm{Nd}-1$, whereas the other three isolates occasionally were able to produce small white blisters. This subtle phenotypic variation suggests further genetic specificity among interactions of Arabidopsis thaliana with different Albugo candida races, potentially associated with additional receptor-like proteins.

Tosa (1992) proposed that interspecific (species-level) variation in innate immunity could be explained in many cases by an accumulation of parasite recognition genes in natural populations of the host that collectively provide broad-spectrum, species-level disease resistance. In other words, susceptible genotypes (lacking any of the existing $R$ genes) are a rare occurrence 
in the host species. Receptor-like genes have been proposed as important determinants of innate immunity in Arabidopsis thaliana to higher taxa (species and subspecies) of parasites from other hosts (Holub 2007). For example, Staal and associates (2006) used transgressive segregation from a recombinant inbred mapping population to identify two TIR-NB-LRR genes that confer resistance to Leptosphaeria maculans (stem canker), an important brassica pathogen that is rarely compatible in wild accessions of Arabidopsis thaliana. WRR4 provides the first analogous example for white rust resistance, which in this case represents a single $R$ gene that can effectively distinguish between different subspecies of the oomycete Albugo candida. The intercross between wild-type Columbia and Ws-3 obviously will provide a more powerful resource for further genetic dissection of non-HR or EDS-independent genes conferring white rust resistance in Columbia Arabidopsis thaliana.

The molecular ecology of plant-microbe interactions represents an exciting frontier in plant biology (Holub 2007). The Arabidopsis thaliana-Albugo candida pathosystem offers a viable model for future research in this area, with leads emerging from the phenomenon of broad-spectrum susceptibility as an important feature of basic compatibility for A. candida in susceptible hosts (Cooper et al. 2008), and the discovery described here of WRR 4 as a determinant of pathogen perception and contributing to species-level resistance in Arabidopsis thaliana. The temporal and spatial distribution of WRR 4 as well as other predicted $R$ genes that enable recognition of Albugo candida races will provide a focus for future research in natural populations of Arabidopsis thaliana.

Characterization of different defense-signaling attributes amongst these $R$ genes will provide an important bridge between the much-needed exploration of Arabidopsis thaliana in its natural habitat and the comparative biochemistry and molecular systems biology research currently being advanced in laboratory research (Holub in press). Investment in Albugo genomics, for example, will be essential to determine whether WRR4 confers avirulence recognition of a highly conserved effector protein that is shared among races 2, 4, 7, and 9 of $\mathrm{Al}$ bugo candida. Given the role of stomata as a preferred site of host entry for bacteria and rust pathogens, it also will be interesting to determine whether A. candida produces functional analogs of bacterial flagellin (i.e., a nonspecific elicitor of innate immunity) or coronatin (i.e., a hormone-like defense suppressor) and, if so, whether this may relate directly to the effector protein from $A$. candida that elicits WRR4-mediated recognition or the sustained broad-spectrum defense suppression induced by virulent isolates of $A$. candida subsp. arabidopsis (Cooper et al. 2008).

Molecular characterization of A. candida effector proteins should be possible because methods have been established for outcrossing A. candida isolates, bioinformatic identification of effector-like genes from oomycetes, and functional testing of oomycete genes via bacterial delivery (Adhikari et al. 2003; Rentel et al. 2008; Sohn et al. 2007; Whisson et al. 2007; Win et al. 2007). Parasite effectors from A. candida will be critical molecular determinants for investigating the relative selective pressures from $A$. candida subsp. arabidopsis and races of $A$. candida derived from other hosts on the innate immunity of Arabidopsis thaliana in natural and experimental populations.

\section{MATERIALS AND METHODS}

\section{Plant and parasite material.}

The standard accessions Col-gll (Col-5), Nd-1, and Ws-3 and the Col-gll $\times \mathrm{Nd}-1$ recombinant inbreds used in this study were deposited in the Nottingham Arabidopsis Stock Centre. We obtained the various defense mutants from corresponding authors of previous publications, including Col-fmol (Bartsch et al. 2006), Col-ndrl (Century et al. 1997), Col-nprl (Cao et al. 1997), Col-pad4 (Glazebrook et al. 1997), Col-rarl/pbs2 (Warren et al. 1999), Col-sidl and sid2 (Nawrath and Metraux 1999), Col-NahG (Delaney et al. 1995), and Ws-eds1-1 (Parker et al. 1996). EMS-treated $\mathrm{M}_{2}$ seed pools of Col-gll was purchased from Lehle Seed and were the source for Colsgtlb (Tör et al. 2002). The fast-neutron-treated $\mathrm{M}_{2}$ seed pools of Col-ndrl were produced at Warwick-HRI.

Acem 2 and Ac9 'Stratford' were collected by E. Holub from their respective host plants found in 1993 at the East Malling Research station in Kent and the author's garden in Stratfordon-Avon (Warwickshire), respectively. Ac2 and Ac7 were isolated by R. Rimmer from B. juncea and B. rapa cultivar evaluation trials in Winnipeg, Manitoba, Canada.

\section{Pathogen handling and inoculations.}

Plant growth, pathogen storage, and inoculation procedures were similar to those described by Holub and associates (1995), with modifications described in detail by Borhan and associates (2001). For experiments in Arabidopsis thaliana, seedlings were grown at 16 to $20^{\circ} \mathrm{C}$ with a 16-h photoperiod at a photon flux density of 80 to $180 \mu \mathrm{mol} \mathrm{m} \mathrm{m}^{-2} \mathrm{~s}^{-1}$. One-week-old seedlings were drop inoculated using a suspension of sporangia (approximately $2 \times 10^{4}$ ) for all the isolates. A sporangia suspension was incubated at 14 to $16^{\circ} \mathrm{C}$ for 2 to $4 \mathrm{~h}$ prior to inoculation and examined on a haemocytometer slide to confirm the release of zoospores. Phenotypes were assessed 7 to 10 dai.

Each of the Albugo candida isolates was maintained on a different susceptible host: Acem2 inoculum was generated on 7to 14-day-old seedlings of either Ws-0 or Ws-eds1; and Ac2, Ac7, and Ac9 were maintained on B. juncea cv. Cutlass, B. rapa cv. Reward, and B. oleracea cv. Maris Kestral, respectively.

\section{Mutant screening.}

Col-gll/wrr4-1 was selected from a screen of approximately 32,000 $\mathrm{M}_{2}$ seedlings (derived from approximately 5,000 $\mathrm{M}_{1}$ plants) that were grown in 24-by-36-cm trays at density of approximately 2,000 seedlings per tray, and inoculated with zoospores when 7 days old. The two fast-neutron mutants were selected from a screen of approximately $20,000 \mathrm{M}_{2}$ seedlings (derived from approximately 3,000 $\mathrm{M}_{1}$ plants).

Seed for T-DNA insertional knockout lines Salk_013292, Salk_131774, and Salk_148037 were obtained from the Arabidopsis Biological Resources Centre. Seedlings, 7 to 10 days old, were inoculated with Ac2 and Ac7. Susceptible lines were grown to mature plants and presence of insert in the WRR4 allele was confirmed by PCR as instructed by the SALK institute. Seed from the selected lines were harvested and used in further pathology tests to confirm the results.

\section{Microscopy observations.}

To detect pathogen and cell death, infected seedlings were stained with trypan blue (Parker et al. 1996) and observed using Differential Interference Contrast (DIC) with a Zeiss Axio Imager Z1 microscope. Alternatively, inoculated seedlings were harvested and placed in microtiter plates containing DAB stain $\left(1 \mathrm{mg} \mathrm{ml}^{-1}\right.$ in $\mathrm{H}_{2} \mathrm{O}, \mathrm{pH} \mathrm{3.8)}$ and returned to the growth rooms for $24 \mathrm{~h}$ (Tör et al. 2002). Seedlings were cleared in chloral hydrate (saturated) for $24 \mathrm{~h}$ and mounted on glass sides in lactoglycerol solution (33\% each of lactic acid, glycerol, and water).

\section{SSLP markers generated} for fine-mapping the WRR4 interval.

NG8 was amplified using the PCR primers 5'-AAGTCACA CCGGTTCATGGT-3' and 5'-TTTCAATTGACTTTTTGTCC 
TTT-3', NG10 was amplified using 5'-AAAGCATGAACCTC ACATGAA-3' and 5'-CCGAGCTGATAAGTGAAACACA-3', and HB-1 was amplified using 5'-TATGACCAAGCTTGTC TC-3' and 5'-TCACTTCTGTCTCTGACG-3'.

\section{Subcloning BAC.}

DNA was prepared from BAC F13N6 and F25P12 using a $\mathrm{CsCl}$ method (Ausubel et al. 1994). DNA was partially digested with Sau3AI and ligated into the binary cosmid vector pSLJ75515 digested with BamHI. Clones were packaged using Gigapack Gold III XL extract (Stratagene, La Jolla, CA, U.S.A.) and transferred into Escherichia coli strain XL1-blue MRA (Stratagene). Bacterial clones were grown on Luria-Bertani (LB) plates containing tetracycline at $12.5 \mu \mathrm{g} \mathrm{ml}^{-1}$, isopropyl- $\beta$-D-thiogalactoside at $20 \mu \mathrm{g} \mathrm{ml}^{-1}$, and X-gal (5-bromo-4chloro-3-indolyl- $\beta$-D-galactoside) at $50 \mu \mathrm{g} \mathrm{ml}^{-1}$. DNA from the cosmid clones was prepared using a standard alkaline lysis procedure. Candidate cosmid clones were identified by PCR.

\section{RNA preparation and reverse-transcriptase PCR.}

RNA was extracted from 2-week-old seedlings of Col-gll using Trizol (Invitrogen, Carlsbad, CA, U.S.A.). Tissue (1 g) was ground in a mortar and pestle containing liquid nitrogen, suspended in $10 \mathrm{ml}$ of Trizol, and left at room temperature for $5 \mathrm{~min}$. After two rounds of purification by chloroform, the supernatant was transferred to a new tube and RNA precipitated by adding $0.6 \mathrm{~V}$ of isopropanol and leaving at room temperature for $20 \mathrm{~min}$. RNA (1 mg) was used to isolate mRNA using Amersham Pharmacia oligo-dt column (Amersham Pharmacia Biotechnology Ltd., Buckinghamshire, U.K.) according to the manufacturer's procedures. A 3' rapid amplification of cDNA ends (RACE) was carried out using RLM_RACE kit (Ambion Inc., Austin, TX, U.S.A.) according to the manufacturer's protocol. The sequence of the gene-specific primer used in 3' RACE was GTGTCGTGATGTCACTTAAC. First-strand cDNA was prepared at $55^{\circ} \mathrm{C}$ from $500 \mathrm{ng}$ of mRNA using thermoscript RT-PCR kit (Invitrogen) and oligo-dT primer. Second-strand cDNA was synthesized using gene-specific primers and performing PCR on $2 \mu \mathrm{l}$ of products of first-strand cDNA with a PCR program of $94^{\circ} \mathrm{C}$ for $4 \mathrm{~min} ; 35$ cycles of $94^{\circ} \mathrm{C}$ for $1 \mathrm{~min}, 55^{\circ} \mathrm{C}$ for $1 \mathrm{~min}$, and $72^{\circ} \mathrm{C}$ for $4 \mathrm{~min}$; and finally $72^{\circ} \mathrm{C}$ for 7 min. WRR4 cDNA was amplified using gene-specific primers containing a $5^{\prime}$ end sequence that was specific for cloning into gateway vectors 3'-ACTTTGTACAAGAAAGCTGGGTTTTC GAATGCTTCTCAGAATACTAGAG, and 5'-TTCGAAGGA GATAGAACCATGGCTTCTTCTTCTTCCTCACCTC.

A 2- $\mu$ l sample of PCR product of WRR4 cDNA, amplified using the above primers, was used as template for a third PCR to complete the attB sites required for cloning into gateway pDONR vectors. The primers used for the third PCR were ATTB1 (forward)-GGGGACAAGTTTGTACAAAAAAGCA GGCTTCGAAGGAGATAGAACC, and ATTB2-(reverse)-GG GGACCACTTTGTACAAGAAAGCTG. The PCR product was gel purified and cloned into the gateway vector pDONR 221 (Invitrogen) as instructed by the manufacturer. WRR4 cDNA insert was sequenced and aligned with the genomic sequence to identify exons and introns.

\section{Sequencing and analysis of WRR4.}

DNA extracted from the cosmid clone P18 using Qiagen midi-prep kit was used as the template for sequencing. The published sequence of At1g56510 was used to design primers from both strands of the gene at intervals of approximately $500 \mathrm{bp}$. DNA sequencing was carried out by the Plant Biotechnology Institute at Saskatoon, Canada. Contiging DNA sequences, prediction and translation of the open reading frame, and alignment of DNA or protein sequence was performed with the Vector NTI
Suite 7 computer program. Domains of WRR4 protein were predicted by Pfam. The programs signalP (version 1.1) and TMpred were used to search for signal peptide and transmembrane regions respectively.

Sequencing of the wild-type and EMS mutant alleles was carried out by first amplifying products using primers generated at intervals of approximately $500 \mathrm{bp}$, then sequencing reactions performed using an ABI Prism Big Dye Terminator cycle sequencing ready reaction kit and an ABI 3100 automated sequencer (Applied Biosystems, Foster City, CA, U.S.A.).

\section{Development of the EDS1 RNAi lines.}

RNAi suppression was used to suppress the function of two EDS1 genes that are present in Columbia Arabidopsis thaliana. A 680-bp fragment from Exon 2 of EDS1 was amplified and cloned into AscI/SwaI sites (sense direction) and SpeI/PacI (antisense direction) of vector pGSA1252 (obtained from ChromDB). Transgenic Col lines were selected and selfed to obtain a single insert homozygous line which was confirmed by segregation of herbicide-resistance marker gene BAR after spraying with DL-phosphinothricin at $100 \mu \mathrm{g} \mathrm{ml}^{-1}$. Primers used to amplify the exon 2 fragments from EDS1 are pGSASpeI/AscI (forward)-TTACTAGTGGCGCGCCCCTCA GGAGGTGCAACTGC, and pGSAPacI/SwaI (reverse)-TTTT AATTAAATTTAAATCCAAGGTCATTGAGCGAAG.

\section{Agrobacterium-mediated transformation of Arabidopsis thaliana.}

Cosmid clones were transferred into Agrobacterium tumefaciens GV3101 by conjugation and grown on LB plates containing tetracycline $\left(12.5 \mu \mathrm{g} \mathrm{ml}^{-1}\right)$ and gentamicin $\left(25 \mu \mathrm{g} \mathrm{ml}^{-}\right.$ $\left.{ }^{1}\right)$. The presence of inserts in cosmid clones was confirmed by PCR on a single clone of transformed Agrobacterium tumefaciens. Ws plants were transformed using the floral dip method (Clough and Bent 1998). Transformants were selected by spraying 7- to 10-day-old seedlings with DL-phosphinothricin at $100 \mu \mathrm{g} \mathrm{ml}^{-1}$.

\section{ACKNOWLEDGMENTS}

Research at Warwick-HRI was supported by funding to E. B. Holub from the U.K. Biotechnology and Biological Sciences Research Council (BBSRC, Agri-foods committee, grant number D16978) and a BBSRC quota award studentship to A. Cooper. Research at Agriculture and Agri-Food Canada was supported by funding to S. R. Rimmer from the Canadian Crop Genomics Initiative. We greatly appreciate the summary data for At1g56510 that was provided by TAIR and the National Science Foundation-sponsored NBS-LRR website. E. B. Holub would like to thank R. Allaby for timely discussions that invigorated the preparation of this manuscript.

\section{LITERATURE CITED}

Aarts, N., Metz, M., Holub, E., Staskawicz, B. J., Daniels, M. J., and Parker, J. E. 1998. Different requirements for EDS1 and NDR1 by disease resistance genes define at least two $R$ gene-mediated signaling pathways in Arabidopsis. Proc. Natl. Acad. Sci. U.S.A. 95:10306-10311.

Adhikari, T. B., Liu, J. Q., Mathur, S., Wu, C. X., and Rimmer, S. R. 2003. Genetic and molecular analyses in crosses of race 2 and race 7 of Albugo candida. Phytopathology 93:959-965.

Ausubel, F. M., Brent, R., Kingston, R. E., Moore, D. D., Seidman, J. G., Smith, J. A., and Struhl, K. 1994. Current Protocols in Molecular Biology. John Wiley and Sons, Inc., New York.

Bartsch, M., Gobbato, E., Bednarek, P., Debey, S., Schultze, J. L., Bautor, J., and Parker, J. E. 2006. Salicylic acid-independent ENHANCED DISEASE SUSCEPTIBILITY1 signaling in Arabidopsis immunity and cell death is regulated by the monooxygenase FMOI and the nudix hydrolase NUDT7. Plant Cell 18:1038-1051.

Bittner-Eddy, P. D., Crute, I. R., Holub, E. B., and Beynon, J. L. 2000. $R P P 13$ is a simple locus in Arabidopsis thaliana for alleles that specify downy mildew resistance to different avirulence determinants in Peronospora parasitica. Plant J. 21:177-188 
Borhan, M. H., Brose, E., Beynon, J. L., and Holub, E. B. 2001. White rust (Albugo candida) resistance loci on three Arabidopsis chromosomes are closely linked to downy mildew (Peronospora parasitica) resistance loci. Mol. Plant Pathol. 2:87-95.

Borhan, M. H., Holub, E. B., Beynon, J. L., Rozwadowski, K., and Rimmer, S. R. 2004. The Arabidopsis TIR-NB-LRR gene RAC1 confers resistance to Albugo candida (white rust) and is dependent on EDS1 but not PAD4. Mol. Plant-Microbe Interact. 7:711-719.

Botella, M. A., Parker, J. E., Frost, L. N., Bittner-Eddy, P. D., Beynon, J. L., Daniels, M. J., Holub, E. B., and Jones, J. D. 1998. Three genes of the Arabidopsis RPP1 complex resistance locus recognize distinct Peronospora parasitica avirulence determinants. Plant Cell 10:1847-1860.

Buell, C. R. 1998. Arabidopsis: A weed leading the field of plant-pathogen interaction. Plant Physiol. Biochem. 36:177-186.

Cao, H., Glazebrook, J., Clarke, J. D., Volko, S., and Dong, X. 1997. The Arabidopsis NPR1 gene that controls systemic acquired resistance encodes a novel protein containing ankyrin repeats. Cell 88:57-63.

Century, K. S., Shapiro, A. D., Repetti, P. P., Dahlbeck, D., Holub, E., and Staskawicz, B. J. 1997. NDR1, a pathogen-induced component required for Arabidopsis disease resistance. Science 278:1963-1965.

Choi, Y.-J., Hong, S.-B., and Shin, H.-D. 2006. Genetic diversity within the Albugo candida complex (Peronosporales, Oomycota) inferred from phylogenetic analysis of ITS rDNA and COX2 mtDNA sequences. Mol. Phylogenet. Evol. 40:400-409.

Clough, S. J., and Bent, A. 1998. Floral dip: A simplified method for Agrobacterium-mediated transformation of Arabidopsis thaliana. Plant J. 16:735-743.

Cooper, A. J., Latunde-Dada, A. O., Woods-Tör, A., Lynn, J., Lucas, J. A., Crute, I. R., and Holub, E. B. 2007. Basic compatibility of Albugo candida in Arabidopsis thaliana and Brassica juncea causes broad-spectrum suppression of innate immunity. Mol. Plant-Microbe-Interact. 21:745-756.

Crute, I., Beynon, J., Dangl, J., Holub, E., Mauch-Mani, B., Slusarenko, A., Staskawicz, B., and Ausubel, F. 1994. Microbial pathogenesis of Arabidopsis. Pages 705-747 in: Arabidopsis. Monograph 27. E. M. Meyerowitz and C. R. Somerville, eds. Cold Spring Harbor Laboratory Press, Cold Spring Harbor, NY, U.S.A.

Delaney, T. P., Friedrich, L., and Ryals, J. A. 1995. Arabidopsis signal transduction mutant defective in chemically and biologically induced disease resistance. Proc. Natl. Acad. Sci. U.S.A. 92:6602-6606.

Feys, B. J., Moisan, L. J., Newman, M. A., and Parker, J. E. 2001. Direct interaction between the Arabidopsis disease resistance signaling proteins, EDS1 and PAD4. EMBO (Eur. Mol. Biol. Organ.) J. 20:5400-5411.

Glazebrook, J. 2001. Genes controlling expression of defense responses in Arabidopsis-2001 status. Curr. Opin. Plant Biol. 4:301-308.

Glazebrook, J., Zook, M., Mert, F., Kagan, I., Rogers, E. E., Crute, I. R., Holub, E. B., Hammerschmidt, R., and Ausubel, F. M. 1997. Phytoalexin-deficient mutants of Arabidopsis reveal that PAD4 encodes a regulatory factor and that four $P A D$ genes contribute to downy mildew resistance. Genetics 146:381-392.

Göker, M., Riethmüller, A., Voglmayr, H., Weiss, M., and Oberwinkler, F. 2004. Phylogeny of Hyaloperonospora based on nuclear ribosomal internal transcribed spacer sequences. Mycol. Progr. 3:83-94.

Gomez-Gomez, L., and Boller, T. 2000. FLS2: An LRR receptor-like kinase involved in the perception of the bacterial elicitor flagellin in Arabidopsis. Mol. Cell 5:1003-1011.

Hammond-Kosack, K. E., and Jones, J. D. 1997. Plant disease resistance genes. Annu. Rev. Plant Physiol. Plant Mol. Biol. 48:575-607.

Holub, E. B. 2001. The arms race is ancient history in Arabidopsis, the wildflower. Nat. Rev. Genet. 2:516-527.

Holub, E. B. 2007. Natural variation in innate immunity of a pioneer species. Curr. Opin. Plant Biol. 10:415-424.

Holub, E. B. Natural history of Arabidopsis thaliana and oomycete symbioses. Eur. J. Plant Pathol. In press.

Holub, E. B., and Beynon, J. L. 1997. Symbiology of mouse ear cress (Arabidopsis thaliana) and oomycetes. Adv. Bot. Res. 24:228-273.

Holub, E. B., Brose, E., Tor, M., Clay, C., Crute, I. R., and Beynon, J. L. 1995. Phenotypic and genotypic variation in the interaction between Arabidopsis thaliana and Albugo candida. Mol. Plant-Microbe Interact. 8:916-928.

Jones, D. A., and Jones, J. D. G. 1997. The role of leucine-rich repeat proteins in plant defences. Adv. Bot. Res. 24:89-167.

Liu, Q., Rimmer, S. R., and Scarth, R. 1989. Histopathology of compatibility and incompatibility between oilseed rape and Albugo candida. Plant Pathol. 38:176-182.

McDowell, J. M., Dhandaydham, M., Long, T. A., Aarts, M. G., Goff, S., Holub, E. B., and Dangl, J. L. 1998. Intragenic recombination and diversifying selection contribute to the evolution of downy mildew resistance at the RPP8 locus of Arabidopsis. Plant Cell 10:1861-1874.

McDowell, J. M., Cuzick, A., Can, C., Beynon, J., Dangl, J. L., and Holub, E. B. 2000. Downy mildew (Peronospora parasitica) resistance genes in Arabidopsis vary in functional requirements for NDR1, EDS1, NPR1 and salicylic acid accumulation. Plant J. 22:523-529.

Mellersh, D. G., and Heath, M. C. 2003. An investigation into the involvement of defense signaling pathways in components of the nonhost resistance of Arabidopsis thaliana to rust fungi also reveals a model system for studying rust fungal compatibility. Mol. Plant-Microbe Interact. 16:398-404.

Melotto, M., Underwood, W., Koczan, J., Nomura, K., and He, S.Y. 2006. Plant stomata function in innate immunity against bacterial invasion. Cell 126:969-980.

Meyers, B. C., Shen, K. A., Rohani, P., Gaut, B. S., and Michelmore, R. W. 1998. Receptor-like genes in the major resistance locus of lettuce are subject to divergent selection. Plant Cell 10:1833-1846.

Meyers, B. C., Kozik, A., Griego, A., Kuany, H., and Michelmore, R. W. 2003. Genome-wide analysis of NBS-LRR-encoding genes in Arabidopsis. Plant Cell 15:809-834.

Nawrath, C., and Metraux, J. P. 1999. Salicylic acid induction-deficient mutants of Arabidopsis express PR-2 and PR-5 and accumulate high levels of camalexin after pathogen inoculation. Plant Cell 11:1393-1404.

Parker, J. E., Holub, E. B., Frost, L. N., Falk, A., Gunn, N. D., and Daniels, M. J. 1996. Characterization of $e d s 1$, a mutation in Arabidopsis suppressing resistance to Peronospora parasitica specified by several different RPP genes. Plant Cell 8:2033-2046.

Peart, J. R., Cook, G., Feys, B. J., Parker, J. E., and Baulcombe, D. C. 2002. An EDS1 orthologue is required for N-mediated resistance against tobacco mosaic virus. Plant J. 29:569-579.

Pound, G. S., and Williams, P. H. 1963. Biological races of Albugo candida. Phytopathology 53:1146-1149.

Rehmany, A. P., Lynn, J. R., Tör, M., Holub, E. B., and Beynon, J. L. 2000. A comparison of Peronospora parasitica (downy mildew) isolates from Arabidopsis thaliana and Brassica oleracea using amplified fragment length polymorphism and internal transcribed spacer 1 sequence analyses. Fungal Genet. Biol. 30:95-103.

Rentel, M. C., Leonelli, L., Dahlbeck, D., Zhao, B., and Staskawicz, B. J. 2008. Recognition of the Hyaloperonospora parasitica effector ATR13 triggers resistance against oomycete, bacterial, and viral pathogens. Proc. Natl. Acad. Sci. U.S.A. 105:1091-1096.

Shapiro, A. D. 2000. Using Arabidopsis mutants to delineate disease resistance signalling pathways. Can. J. Plant Pathol. 22:199-216.

Slusarenko, A. J., and Schlaich, N. L. 2003. Downy mildew of Arabidopsis thaliana caused by Hyaloperonospora parasitica (formerly Peronospora parasitica). Mol. Plant Pathol. 4:159-170.

Sohn, K H, Lei, R, Nemri, A, and Jones, J. D. 2007. The downy mildew effector proteins ATR1 and ATR13 promote disease susceptibility in Arabidopsis thaliana. Plant Cell. 19:4077-4090.

Song, J., Bradeen, J. M., Naess, S. K., Raasch, J. A., Wielgus, S. M., Haberlach, G. T., Liu, J., Kuang, H., Austin-Philips, S., Buell, C. R., Helgeson, J. P., and Jiang, J. 2003. Gene RB cloned from Solanum bulbocastanum confers broad spectrum resistance to potato late blight. Proc. Natl. Acad. Sci. U.S.A. 100:9128-9133.

Staal, J., Kaliff, M., Bohman, S., and Dixelius, C. 2006. Transgressive segregation reveals two Arabidopsis TIR-NB-LRR resistance genes effective against Leptosphaeria maculans, causal agent of blackleg disease. Plant J. 46:218-230.

Tai, T. H., Dahlbeck, D., Clark, E. T., Gajiwala, P., Pasion, R., Whalen, M C., Stall, R. E., and Staskawicz, B. J. 1999. Expression of the Bs2 pepper gene confers resistance to bacterial spot disease in tomato. Proc. Natl. Acad. Sci. U.S.A. 96:14153-14158.

Tan, X., Meyers, B. C., Kozik, A., West, M. A. L., Morgante, M., St. Clair, D. A., Bent, A. F., and Michelmore, R. W. 2007. Global expression analysis of nucleotide binding site-leucine rich repeat-encoding and related genes in Arabidopsis. BMC Plant Biol. 7:56. Published online.

Tör, M., Gordon, P., Cuzick, A., Eulgem, T., Sinapidou, E., Mert-Türk, F., Can, C., Dangl, J. L., and Holub, E. B. 2002. Arabidopsis SGT1b is required for defense signaling conferred by several downy mildew resistance genes. Plant Cell 14:993-1003.

Tosa, Y. 1992. A model for the evolution of formae speciales and races. Phytopathology 82:728-729.

Underwood, W. Melotto, M., and He, S. Y. 2007. Role of plant stomata in bacterial invasion. Cell Microbiol. 9:1621-1629.

Verma, P. R., Harding, H., Petrie, G. A., and Williams, P. H. 1975. Infection and temporal development of mycelium of Albugo candida in cotyledon of four Brassica species. Can. J. Bot. 53:1016-1020.

Voglmayr, M., and Riethmüller, A. 2006. Phylogenetic relationships of Albugo candida (white blister rusts) based on LSU rDNA sequence and oospore data. Mycol. Res. 110:75-85.

Warren, R. F., Merritt, P. M., Holub, E. B., and Innes, R. W. 1999. Identification of three putative signal transduction genes involved in $R$ genespecified disease resistance in Arabidopsis. Genetics 152:401-412.

Whisson, S. C., Boevink, P. C., Moleleki, L., Avrova, A. O., Morales, J. 
G., Gilroy, E. M., Armstrong, M. R., Grouffaud, S., van West, P., Chapman, S., Hein, I., Toth, I. K., Pritchard, L., and Birch, P. R. 2007. A translocation signal for delivery of oomycete effector proteins into host plant cells. Nature 450:115-118.

Wiermer, M., Feys, B. J., and Parker, J. E. 2005. Plant immunity: The EDS1 regulatory node. Curr. Opin. Plant Biol. 8:383-389.

Win, J., Morgan, W., Bos, J., Krasileva, K. V., Cano, L. M., ChaparroGarcia, A., Ammar, R., Staskawicz, B. J., and Kamoun, S. 2007. Adaptive evolution has targeted the C-terminal domain of the RxLR effectors of plant pathogenic oomycetes. Plant Cell 19:2349-2369.

Zipfel, C., Robatzek, S., Navarro, L., Oakeley, E. J., Jones, J. D., Felix, G., and Boller, T. 2004. Bacterial disease resistance in Arabidopsis through flagellin perception. Nature 428:764-767.
AUTHOR-RECOMMENDED INTERNET RESOURCES

Nottingham Arabidopsis Stock Centre website: nasc.nott.ac.uk/ Lehle Seed Arabidopsis information resource: www.arabidopsis.com The Arabidopsis Information Resource (TAIR): www.arabidopsis.org/ Wellcome Trust Sanger Institute Pfam website: www.sanger.ac.uk/Software/Pfam/index.shtml

Center for Biological Sequence Analysis SignalP server: www.cbs.dtu.dk/services/SignalP/

Swiss Institute of Bioinformatics EMBnet Tmpred website: www.ch.embnet.org/software/TMPRED_form.html

National Science Foundation-sponsored NBS-LRR link: ag.udel.edu/meyers_lab/data.htm 